\title{
Routing Strategy and Quality of Service (QoS) Realization Research of Guangxi Meteorology Network
}

\author{
ZHANG Jun ${ }^{1, a}$, PENG Fuxiang ${ }^{1, b}$ \\ ${ }^{1}$ Beihai Meteorological Administration, No.66, Guangdong road, Beihai, Guangxi, 536000, China \\ akwpjun@yahoo.cn, bpengfuxiangbh@sina.cn
}

\begin{abstract}
Keywords: communication network, border gateway protocol (BGP), open shortest path first interior gateway protocol (OSPF), routing strategy
\end{abstract}

\begin{abstract}
Large cover area and long distance transmission are main characteristics of a meteorological communication network. A satellite communication technology has been mainly adopted in the field of meteorological communication for a long time, because an optical communication technology has become the important support technology during a new network transformation, meteorological departments establish a nationwide meteorological broadband network. The paper aims at the structure of the Guangxi meteorological broadband network, points out how to use a routing strategy which combines a border gateway protocol (BGP) with an open shortest path first interior gateway protocol (OSPF) to solve the problem of entire network routing flapping and provides a basic method to dynamically realize the quality of service (QoS) guarantee of various kinds of service data in the meteorological broadband communication network by combining meteorological data services and class based queueing (CBQ).
\end{abstract}

\section{Introduction}

With the construction of a national meteorological broadband communication network system, a meteorological broadband communication network has become the main platform of a new generation meteorological communication network, acquires meteorological data such as meteorological observation data, radar data, atmospheric sounding data and the like, and transmits various meteorological materials such as numerical forecast product, satellite product, radar product and the like, and assists multi-media transmission services such as television video conference and the like and a office automation system. The time used for constructing the Guangxi meteorological broadband communication network is nearly ten years, and the Guangxi meteorological broadband communication network meets the requirement of various kinds of new observation material transmission, the requirement of information sharing platform construction and the requirement on network of new service, solves the problem of aging of the Guangxi meteorological satellite communication system, and indicates the Guangxi meteorological communication network enters a new development stage. However, the meteorological broadband network has the advantages of a wide coverage area and long transmission distance, but also has the fundamental problems that how to solve routing flapping in long distance broadband network and bandwidth allocation of the whole network namely ensuring quality of service (QoS).

\section{Meteorological broadband network architecture}

The requirement variation of the meteorological service and the development of a communication network technology are the main driving force for meteorological information network system structure evolution. The conventional network system structure has the disadvantages of a small bandwidth, a slow speed, system aging and no capability of integrated service data transmission. With a large amount of optical fiber applied in a wide area network, a metropolitan area network and the backbone of an access network, the popularization and application of an optical network are irreversible.

With the background above, the meteorological broadband communication network which has been used is utilized as the main component of the next generation China meteorological administration national communication network system, combined with a current China 
meteorological national satellite communication network system, provides the transmission capability among a Beijing center, an area center and a provincial system, supports the comprehensive transmission of data, video and voice, and provides various kinds of service quality assurance. The national meteorological broadband network connection logical topological graph is shown as Fig. 1. China meteorological administration comprehensively compares a plurality of communication technologies such as synchronous digital hierarchy (SDH), an asynchronous transfer mode (ATM), multi-protocol label switch (MPLS), virtual private network (VPN) and the like, and selects an SDH digital line and an MPLS VPN network as the data transmission technology of provincial cities to Beijing. ${ }^{[1]}$

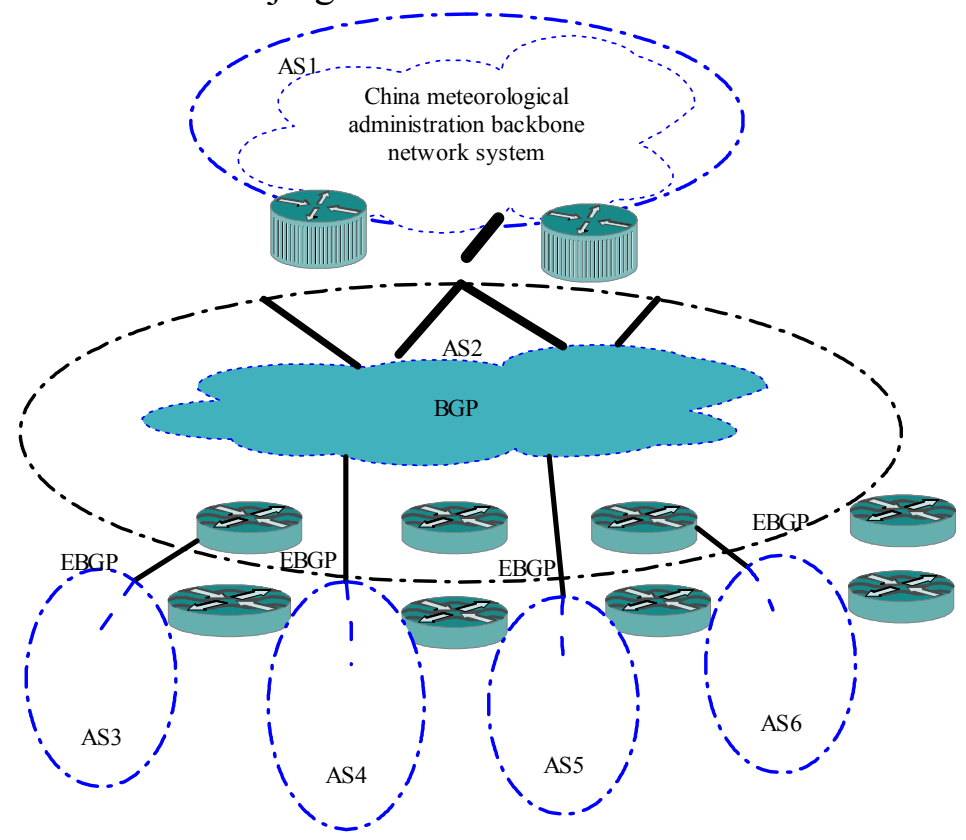

Fig. 1 China meteorological administration broadband network connection logic topological graph

A routing system is the core component of network interconnection and controls the transmission of network flow. An unstable router can cause network performance reduction and results in a certain extent network connection interrupt. ${ }^{[2]}$ An open shortest path first (OSPF) and a border gateway protocol (BGP) are adopted on the routing design of the Guangxi meteorological broadband communication network, and a quality of service (QoS) of the combination of service classify and service transmission requirements is established.

\section{Routing protocol}

\section{Interior gateway protocols-open shortest path first (IGP-OSPF) protocol}

The OSPF which is provided by Internet engineering task force (IETF) is an IGP based on link states. The optimization of the IGP-OSPF protocol requires to be respectively configured and realized on network equipment such as a service router and a broadband accessing server on the service control layer of the metropolitan area network, a core converge server on the core converge layer of the metropolitan area network, a core router and all network equipment supporting optimizing characteristics on the core layer of the metropolitan area network. An intelligent timer technology is introduced, and the timer can dynamically change time interval according to a preset configured parameter and an index avoidance rule. ${ }^{[3]}$

\section{Border gateway protocol (BGP)}

The BGP is a mature routing protocol in a current network. ${ }^{[4][5]}$ The BGP maintains a routing list, transmits routing update information and determines the routing based on routing metric. The BGP is the routing protocol among autonomous systems and is an external gateway protocol, wherein the autonomous system is a system consisting of a group of networks and gateways controlled by a single manager, and the autonomous system can be regarded as a set of a host computer, a server and other network equipment managed by the same entity or enterprise. The main function of the BGP is that network accessible information is changed among systems using the BGP protocol. ${ }^{[6]}$ 
In addition, the realization of quality of a service (QoS) safeguard mechanism is very necessary on the Guangxi meteorological network system for ensuring the service quality of real-time services and meeting user requirement and the requirement of dividing various kinds of services in the system. In practical application, the implementation of effective QoS control in a large scale network is difficult, and the main reason is as following that: the QoS relates to many complex problems such as QoS routing, admission control and network policy management and the like; and many self-control systems on the Internet result that the coordination among various systems is difficult. In the paper, the QoS is designed and realized for the meteorological network.

Aiming at the Guangxi meteorological network system, the paper presents the routing design and the QoS design in detail as follows.

\section{Key technologies}

\section{Routing strategy}

The rule of the routing plan and the routing design is easily to be arranged and maintained on the premise of meeting service requirement. Although the broadband communication network can arrange static routing, the setting of a large amount of static routing can cause a management and maintenance disaster, and the human configuration errors can occur easily. Simultaneously, how to avoid the unstable network caused by routing flapping in the long distant broadband network is also a problem. The ODPF is adopted as an IGP in the Guangxi meteorological broadband communication network system for solving the problems above. The ebgp is adopted to connect with the external network for ensuring the connection with a national meteorological information center, ensuring the independence of each local router and the clarity of lay, and reducing the routing flapping. The node equipment of each level connects with local level 2 network by static routing, because if the provincial level is used as one area of ospf, the change of any province routing can be transmitted to the broadband backbone network and the area of other provinces.

The OSPF protocol is used for the interior router of the broadband network router to ensure the accessibility of the BGP routing, and the local area network and service routing are forbidden to be added into the IGP to ensure the stability of the network.

(1) IGP routing design

The OSPF is adopted as the IGP in the system. The OSPF is in charge of publishing the routing of all link addresses and the routing of the loopback address of equipment. The BGP publishes all service network routing and local area network routing. The node router of a meteorological communication master station, the interconnection interface of a provincial node router, interfaces among master station node routers and backbone equipment loopback interface loopback0 are unified arranged in a backbone area. The process-id of the OSPF is the local concept of a router, and cannot influence other router. The process-ids of the OSPF of all router and core 3-layer switchboard are set into one value for the unity and standard of the whole network system.

(2) BGP routing design

The meteorological communication master station connects with the provincial nodes by an ebgp routing protocol, and the two routers of the master station connects with each other by an ibgp protocol. The provincial nodes can learn the routing information of the whole network by bgp routing strategy, and routing learning and information exchange of different provincial nodes can be limited by the routing strategy. The method comprises the following steps of: adopting a private AS number, and establishing ebgp neighborhood relationship between provincial node routers and a center. The rule of the method is that the routing protocol of the interior local area network of a second level network is not changed, and the local area network and the service router are transmitted by BGP in the broadband network, and does not enter the IGP. Simultaneously, the routing of all service addresses and local area network addresses is published by BGP for reducing the influence of the network line flapping on the whole network routing. The network command used in the BGP in the second level network publishes the service network section merge routing by the network command of the BGP, or the interior network and service routing are published in the $\mathrm{BGP}$ to realize by a reconfiguration command. The local area network routing and service routing 
of the provincial nodes are published by provincial routing equipment, and the local area network and service routing of the China meteorological administration are realized by master station node routing equipment. In addition, because the different exchange visit conditions among provinces, the control of the exchange visit among provinces is realized in two ways as following.

1) producing an access control list on the NE08E router of each provincial node, filtering data packets by permit and deny statements to realize the exchange visit control among provinces;

2) performing the BGP routing information control on a center node, filtering the data packets by the BGP routing information published by peer to make each province only can learn the required BGP routing, and the exchange visit control among the provinces is realized.

\section{QoS design}

The meteorological broadband networks all over the China can meet video, voice and data transmission on the overall design, the QoS is required to be ensured in multi-media communication. Network equipment which can offer abundant QoS mechanism in the broadband network construction, a reasonable and scientific QoS routing strategy is designed according to the characteristics of the meteorological services, the particular implementation strategy is as following:

(1) marking the different services respectively using IP priority or a differentiated services code point (DSCP), and classifying the flow on the basis of the IP priority or DSCP, and performing CAR speed limit focusing certain services as required;

(2) QoS performances such as bandwidth, time delay, time delay jitter and the like can be ensured of the different services on the output interface of the master station and provincial node router by class based on queueing (CBQ) scheduling algorithm.

The QoS guarantee of various kinds of service data of the new generation meteorological broadband communication network can be dynamically realized by the $\mathrm{CBQ}$, the following examples are taken for explanation.

At present, the common data types in meteorological departments are general office automatic (OA) data, radar observation material and resource data and video data of automatic observation stations.

The video data is special, the time delay, jitter, and packet loss of the video data have strict requirements, so the video data is allocated to low latency queueing (LLQ) having the highest priority. If a message is in LLQ, the message in the LLQ is always be transmitted until no message is in the LLQ, or the maximum reserved bandwidth allocated for the LLQ is exceed, then messages in other queues are scheduled and transmitted. When a jam is happened, information management should be fully guaranteed, so a simple network management protocol and OSPF routing information are classified to the LLQ queue.

Because dispersed ftp services are required to be realized in the provincial administration and national administration, each ftp service should be performed with individual service port defining. An SUSE system used by the server can realize allocating a service port for each ftp service independently, the input ports of the provincial and national administration are adopted to classify tcp ports, and corresponding marks are given, the different queues are defined according to the marks on output ports, and because each queue has defined corresponding strategy, the QoS division of $\mathrm{ftp}$ services, video NOTES services and the like is realized. However, the 21 port defaulted by the system cannot be adopted for the ftp service port number, users using windows operating system must using particular ftp software to perform transmission.

\section{Conclusion}

The establishment of the new generation meteorological transmission network can effectively solve the problems of failure display of the current satellite communication system, system volume shortage, difficulties for expanding the system volume and the like, and meet the requirement of the development of the meteorological service, particularly the requirement of data transmission, and really realize a three-in-one multi-media communication. ${ }^{[7]}$ According to the paper, the reasonable 
routing strategy and the QoS routing design ensure the stability and reliability of the whole network, and the condition of the routing flapping generated by transmitting the provincial routing change to the whole network can be avoided.

\section{Reference}

[1] China meteorological broadband network system architecture research [J]. Lang Hongliang.Beijing: Meteorological Science and Technology, 2009, 09, Vol. 34, supply.

[2] BGP routing stability mornitoring tool design based on routing-refresh [J]. Li Wei, Zhang Dafang. Beijing: Computer Engineering and Applications, 2010, 46 (8) 75-77.

[3] IGP-OSPF routing protocol network optimization technology [J]. Yao Linyan. Beijing: Network and Communication, 2012, 1-2.

[4] TCP/IP routing technology [M]. Doyle, Carroll. Ge Jianli, Wu Jianzhang. Beijing: Post and Telecom press, 2007.

[5] Rekhter Y, Li T, Hares S.IETF RFC 4271 A Border Gateway Protocol (BGP-4) [S]. Reston: Internet Society,2006.

[6] Border Gateway protocol (BGP) security analysis and enhance [J]. Ji Li. Beijing: Computer Application and Software, 2007, vol. 24, No.3.

[7] New generation of esoscale automatic weather station network monitoring system [J]. Wu Naijun, $\mathrm{Hu}$ Yufeng, Li Jia. Beijing: Meteorological Science and Technology,2005,Oct.Vol.33,No.5. 